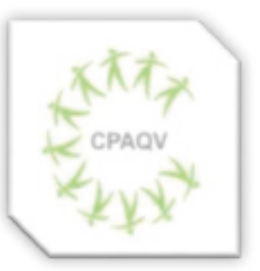

ISSN: 2178-7514

Vol. 12| No. 1| Ano 2020
ARTIGO ORIGINAL

\section{CAPACIDADES FÍSICAS E PERFIL ANTROPOMÉTRICO DE CRIANÇAS E ADOLESCENTES PRATICANTES REGULARES DE ATIVIDADES RECREATIVAS}

Physical Capabilities and Anthropometric Profile of Children and Adolescents Regularly Practicing Recreational Activities

\author{
Francisco Isaquiel Gomes da Silva ${ }^{1,5}$, Robson Salviano de Matos ${ }^{2}$, \\ José Messias Vieira Marques Filho², Marília Porto Oliveira Nunes ${ }^{3}$, Felipe Rocha Alves ${ }^{2}$, \\ Evanice Avelino de Souza ${ }^{4}$, Júlio César Chaves Nunes Filho ${ }^{1,2}$
}

\begin{abstract}
RESUMO
Compreender a importância do lúdico para a vida de crianças e adolescentes se faz muito necessário. O brincar pode ser usado como uma ferramenta para o desenvolvimento humano. Além disso, os jogos recreativos podem ser utilizados como uma estratégia fundamental para o controle da massa corporal e manutenção da saúde. Este estudo tem o objetivo de analisar as capacidades físicas e o perfil antropométrico de crianças e adolescentes praticantes de atividades recreativas. Trata-se de um estudo descritivo, de corte transversal e abordagem quantitativa. A amostra foi de 50 indivíduos, com idades entre 08 e 16 anos, atendidos por um projeto social na cidade de Cascavel, CE. Usou-se como instrumento de medida testes para avaliar capacidades físicas (velocidade de $20 \mathrm{~m}$ e agilidade do quadrado), cálculo do índice de massa corporal (IMC) e relação cintura-estatura (RCE). Os resultados revelaram quando comparado os dados antropométricos e idade entre os sexos não foi verificado diferença estatisticamente significativa, assim como para os valores médios dos testes velocidade e agilidade. Houve associação do risco de desenvolvimento de doenças cardiovasculares, a partir do RCE, com os sexos $(\mathrm{p}=0,03)$. Por fim, a associação entre o gênero e as classificações dos testes de agilidade e velocidade não se apresentaram significativas. Concluiu-se que entre as crianças e adolescentes participantes do projeto "iniciação" não houve diferenças significativas quando comparado as capacidades físicas (velocidade e agilidade) e o perfil antropométrico. Entretanto, constatou-se um alto risco para o desenvolvimento de doenças cardiácas, tendo como parametro o RCE, para o sexo feminino.
\end{abstract}

Palavras-chave: Recreação. Aptidão física. Criança. Adolescente. Saúde.

\begin{abstract}
Understand the importance of playfulness to the lives of children and adolescents is very necessary. The play can be used as a tool for human development. In addition, recreational games can be used as a key strategy for the control of body weight and maintaining health. This study aims to analyze the physical abilities and anthropometric profile of children and adolescents practitioners of recreational activities. It is a descriptive study, cross-sectional and quantitative approach. The sample consisted of 50 subjects, aged from 08 to 16, attended by a social project in the city of Cascavel, CE. It was used as a measuring instrument tests to evaluate physical capabilities (20m square of speed and agility) calculate body mass index (BMI) and waist-to-height ratio (WtHR). The results showed compared anthropometric data gender and age was not observed a statistically significant difference, as well as the average values of the tests speed and agility. There was an association of the risk of developing cardiovascular diseases, from the CER, to gender $(p=0.03)$. Finally, the association between gender and the ratings of agility and speed tests did not show significant. It was concluded that among children and adolescents participating in the project "initiation" there were no significant differences when compared to the physical abilities (speed and agility) and the anthropometric profile. However, there was a high risk for developing heart disease.
\end{abstract}

Keywords: Elderly; Institutionalization; Geriatric physiotherapy.

Autor de correspondência
1 Instituto Dom José de Educação e Cultura (IDJ)
2 Universidade Federal do Ceará (UFC)
3 Universidade de Fortaleza (UNIFOR)
4 Faculdade de Tecnologia do Nordeste (FATENE)
5 Universidade Vale do Acaraú (UVA)

José Messias Vieira Marques Filho,

Av. da Universidade, 2357, Benfica. CEP: 60020-180.

DOI: doi.org/10.36692/cpaqv-v12n1-18

E-mail: messias.filho.ef@gmail.com 


\section{INTRODUÇÃO}

O desenvolvimento humano é um processo de formação progressiva que ocorre ao longo da vida. Este processo contempla aspectos relacionados aos domínios motores, cognitivos, afetivos e físicos, ou seja, ao se falar em desenvolvimento humano, deve-se analisar todo o processo de forma holística. Através de estímulos, a criança inicia seu desenvolvimento da coordenação motora grossa e explora seu corpo de forma gradativa ${ }^{(1)}$.

O desenvolvimento motor diz respeito, mais especificamente, as mudanças relacionadas ao padrão de movimento observado ao longo da vida. Esta capacidade sofre alterações, no transcorrer da vida, devido a características do indivíduo (crescimento, maturação e capacidade física), do ambiente (espaços, superfícies, socioculturais) e da tarefa (objetivos, as regras e equipamentos). O desenvolvimento vai se aprimorando ao passar dos anos, com estimulações, um ambiente favorável ao seu crescimento e com interação da família e da comunidade ${ }^{(1)}$.

Estas mudanças ocorrem ao longo das fases de desenvolvimento (fase motora reflexiva, rudimentar, fundamental $\mathrm{e}$ especializada) e seus estágios (estágio de pré-controle, inicial, elementar, maduro, transitório, de aplicação, dentre outros). Contudo, sabe-se que pode haver diferenças individuais e que a idade cronológica não é o único fator influenciador do nível de desenvolvimento. Se as crianças passarem pelo período crítico para o desenvolvimento das habilidades motoras sem conseguir aprimorá-las até o padrão maduro, podem ter maiores dificuldade no processo de aquisição do padrão motor posteriormente. Portanto, o próprio desenvolvimento da fase motora especializada pode ser prejudicado ${ }^{(2)}$.

Nas brincadeiras as crianças desenvolvem capacidades importantes, tais como a atenção, a imitação, a memória e a imaginação ${ }^{(3)}$. Ramos ${ }^{(4)}$, descreve que a interação com o brincar favorece as relações de autonomia, a criança assume um papel favorável para o seu aprendizado reconhecendo os brinquedos e socializando com outras crianças. É através de brincadeiras e jogos que a criança aprende a agir, sua curiosidade é estimulada, adquiri iniciativa e autoconfiança, proporciona o desenvolvimento da linguagem, do pensamento e da concentração.

Sabe-se que o jogo recreativo é essencial na promoção da saúde de crianças e adolescentes com sobrepeso e obesidade ${ }^{(5)}$. Entretanto, evidências acumuladas indicam que $o$ nível de insuficiência de atividade física ou sedentarismo entre as crianças e adolescentes são alarmantes a nível mundial ${ }^{(6,7)}$.

Em uma análise conjunta usando um levantamento de dados de 298 pesquisas com base nas escolas de 146 países, territórios e áreas, incluindo 1,6 milhões de alunos com idades entre 11-17 anos entre o período de 2001 a 2016, constatou que globalmente, em 
2016, 81,0\% de alunos entre 11-17 anos eram insuficientemente ativos fisicamente, sendo $77,6 \%$ meninos e $84,7 \%$ meninas ${ }^{(7)}$.

Uma revisão sistemática recente descobriu que as crianças pré-escolares (cerca de 3 a 5 anos) passam até 12 horas por dia em comportamento sedentário. Além disso, o mesmo estudo afirma que as crianças pré-escolares gastam em média duas horas por dia em tempo de tela, descumprindo as recomendações atuais que são de menos de uma hora de tempo de tela por $\mathrm{dia}^{(6)}$.

Importante constatar que comportamentos relacionados à saúde, como atividade física(AF) e comportamento sedentário(SED), são tipicamente estabelecidos durante a primeira infância. Evidências sugerem que esses comportamentos acompanham o tempo. Como os níveis de AF diminuem com a idade em crianças e adolescentes em idade escolar, os anos pré-escolares são fundamentais para garantir níveis suficientes de AF para a saúde e o desenvolvimento normal ${ }^{(8)}$.

Portanto, percebe-se que o lúdico é extremamente importante como instrumento de educação e saúde e profundamente rico, uma vez que transfere incontáveis proveitos para a promoção da criança, seja ele cognitivo, motor, social e afetivo. Dessa forma é através do jogo que a criança constrói, concebe, vivencia e adquire habilidades. $\mathrm{O}$ brincar promove vivências prazerosas e dar o ser comportamentos e assimilação de surgimentos de regras sociais, bem como proporciona também no desenvolvimento do entendimento do ser humano, colaborando com que suas sensações se tornem clara, promovendo enriquecimento na vida central da criança. As atividades lúdicas propiciam realmente um senso de responsabilidade e as crianças aprendem o valor de socializar um com outro, pois a criança e jovem aprende melhor brincando e muitos conteúdos podem ser ensinados por meio das brincadeiras, as atividades com jogos ou brinquedos podem ter objetivos didáticos pedagógicos que visem proporcionar o desenvolvimento integral do educando ${ }^{(4)}$.

Sendo assim, este estudo tem o objetivo de analisar as capacidades físicas e o perfil antropométrico de crianças e adolescentes praticantes de atividades recreativas.

\section{MATERIAL E MÉTODOS}

\section{Tipo de estudo}

O presente estudo caracterizouse como uma pesquisa descritiva, de corte transversal e abordagem quantitativa.

\section{Local e período}

A pesquisa foi realizada no projeto intitulado "iniciação". Trata-se de uma atividade social focado no desenvolvendo de atividades recreativas e lazer com crianças e adolescentes. $\mathrm{O}$ projeto acorre na cidade de Cascavel-CE, região metropolitana de 
Fortaleza, e funciona em três núcleos: Universidade Vale do Acaraú (UVA), Escola de Ensino Fundamental Luís Pacheco do Amaral e no Centro de atendimento psicossocial (CAPS). O estudo foi realizado no período de setembro e outubro de 2019.

\section{Participantes}

A população do estudo foi formada por 150 crianças e adolescentes, de ambos os sexos e com faixa etária entre 08 e 16 anos. A amostra final foi de 50 indivíduos, sendo 38 do sexo masculino e 12 do sexo feminino.

Foram incluídos na pesquisa somente os voluntários matriculados regularmente em uma escola. Como critérios de exclusão foram adotados: alunos em que se recusaram a realizar os testes propostos de velocidade e agilidade, bem como a coleta dos dados antropométricos.

O estudo obteve aprovação do Comitê de Ética em Pesquisa com Seres Humanos da Universidade Estadual do Ceará (UECE) sob parecer de no 1.430.345 CEP/UECE. Os voluntários e os responsáveis assinaram o Termo de Consentimento Livre e Esclarecido (TCLE).

\section{Delineamento experimental}

A coleta dos dados foi executada em duas etapas. $\mathrm{Na}$ primeira, foram coletados os dados antropométricos: peso corporal, estatura e circunferência da cintura (CC). Após isso, foi calculado o índice de massa corporal
(IMC) (peso corporal/estatura2) e relação cintura-estatura (RCE) (circunferência da cintura/estatura). De acordo com a equação do IMC, os participantes foram classificados qualitativamente em três categorias: magro ou baixo peso $(<18,5 \mathrm{~kg} / \mathrm{m} 2)$; normal ou eutrófico (IMC 18,5-24,9 kg/m2), sobrepeso (IMC 25,0- 29,9 kg/ m2)9. Foi utilizado o ponto de corte $\geq 0,50$ como maior risco na RCE ${ }^{(10)}$.

No segundo momento, foi realizado dois testes (velocidade e agilidade) de desenvolvimento das capacidades motoras ${ }^{(11)}$. A velocidade é definida como a capacidade de realizar um movimento no menor espaço de tempo. Para a aplicação do teste foi utilizado: cronômetro e um espaço de $20 \mathrm{~m}$ demarcado com três linhas paralelas no solo da seguinte forma: a primeira (linha de partida); a segunda, distante $20 \mathrm{~m}$ da primeira e a terceira linha, sendo marcada a um metro da segunda (linha de chegada). A terceira linha serve como referência de chegada para o aluno na tentativa de evitar que ele inicie a desaceleração antes de cruzar a linha de cronometragem. Foram utilizados cones para a sinalização das linhas. O cronômetro foi travado quando o aluno ao cruzar a segunda linha (linha de cronometragem) tocar pela primeira vez ao solo. Foi registrado o tempo do percurso em segundos e centésimos de segundos (duas casas após a vírgula). $\mathrm{O}$ Quadro 01 demonstra a classificação do teste de velocidade. 


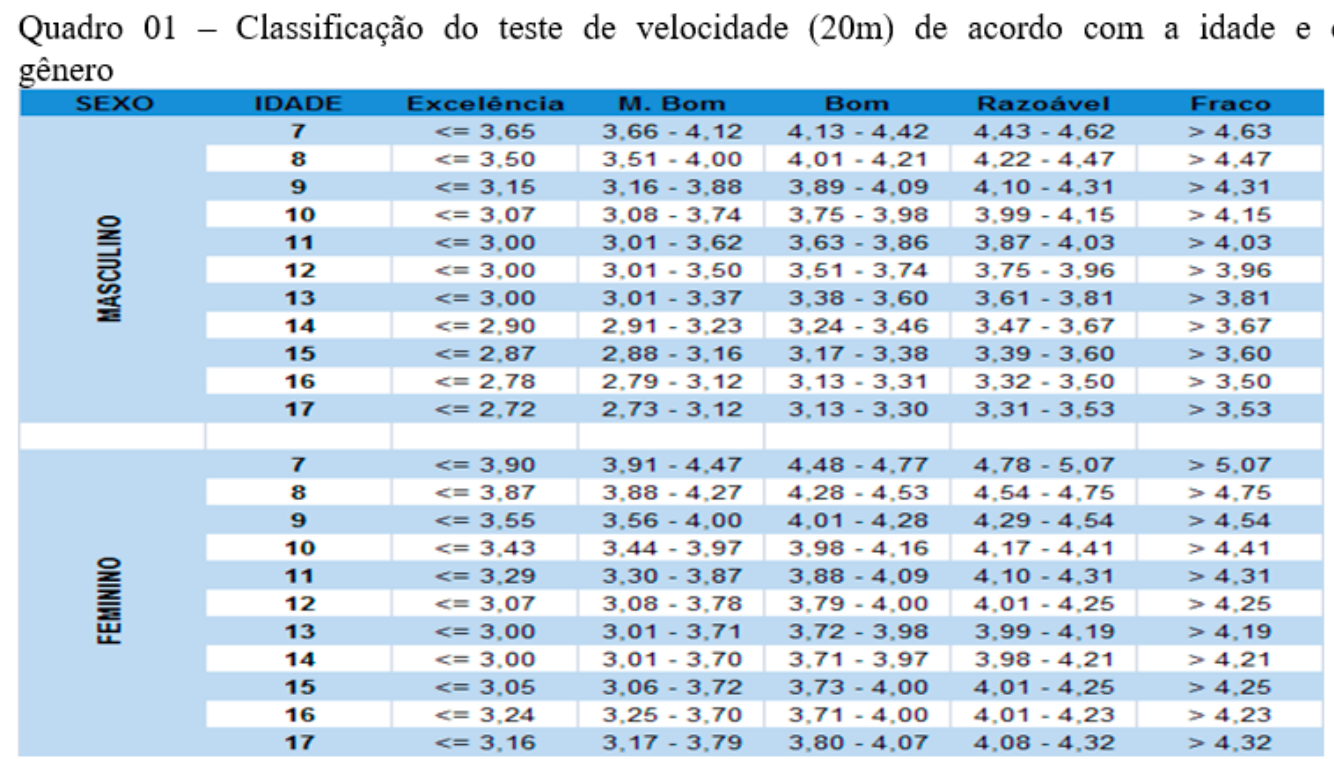

Fonte: Manual do Projeto Esporte Brasil

A agilidade, que é definida como a da linha de partida. Após sinal do avaliador, capacidade de coordenar os movimentos de eles se deslocavam até o próximo cone em uma forma rápida e precisa, foi avaliada a direção diagonal ${ }^{(1)}$, depois iam em direção partir do teste do quadrado, onde, na quadra ao cone situado a esquerda ${ }^{(2)}$, depois foi desenhado com giz um quadrado com novamente se deslocavam para o terceiro quatro metros de lado e quatro cones de cone em diagonal ${ }^{(3)}$, e por fim, retornavam $50 \mathrm{~cm}$ cada distribuídos nas pontas desse a direita ao último cone correspondente ao desenho. Os alunos partiam da posição ponto de partida ${ }^{(4)}$. A classificação do teste ereta e com um pé avançado à frente atrás de agilidade encontra-se no Quadro 02.

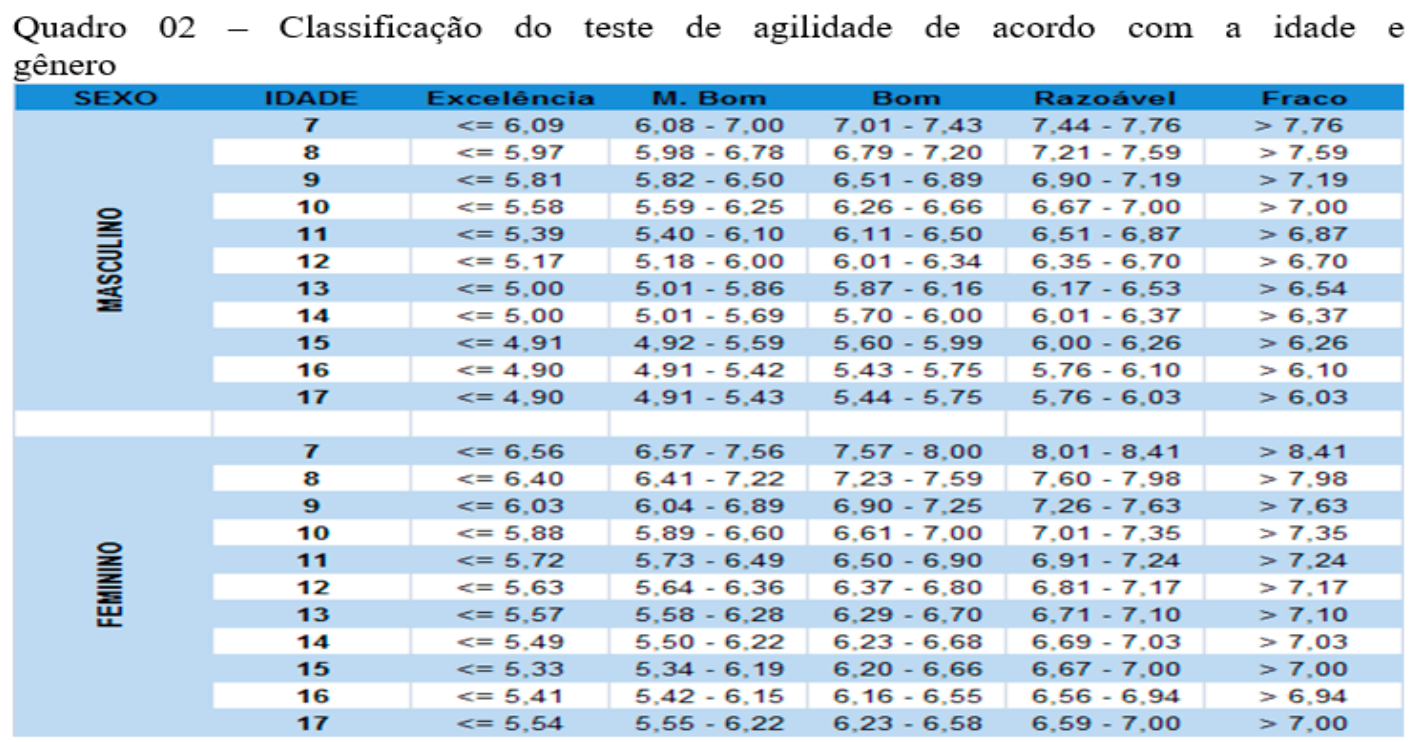

Fonte: Manual do Projeto Esporte Brasil 


\section{Análise estatística}

Inicialmente, foi realizada a análise descritiva da amostra. Posteriormente, foi utilizado o teste de amostras independentes para comparação das variáveis entre os sexos e o teste Qui-quadrado para associação das variáveis. A significância estatística foi de $\mathrm{p}<0,05$.

\section{RESULTADOS}

Foram analisadas 50 crianças e idade 11,62 ( \pm 0,13 anos), peso 44,46 $( \pm 13,97$ $\mathrm{kg})$, estatura 1,51 ( \pm 0,14 m), IMC 18,54 ( \pm 3,62 $\mathrm{kg} / \mathrm{m} 2)$, circunferência da cintura $72,8( \pm 12,1$ cm) e Relação Cintura Estatura de 0,48 ( \pm 0,08).

Do total da amostra, a maioria (76\%; $\mathrm{n}=38$ ) foi composta pelo sexo masculino. Quando comparado os dados antropométricos e idade entre o sexo masculino e feminino, não foi verificado diferença estatisticamente significativa ( $p>0,05)$, demostrado na Tabela 01.

adolescentes de ambos os sexos, com medias de:

Tabela 01: Comparação das variáveis antropométricas e idade entre os sexos.

\begin{tabular}{llllll}
\hline & \multicolumn{2}{c}{ MASCULINO (N=38) } & \multicolumn{3}{c}{ FEMININO (N=12) } \\
& M & DP & M & DP & P \\
\hline PESO & 43,12 & $\pm 14,76$ & 47,86 & $\pm 10,85$ & 0,311 \\
ALTURA & 1,51 & $\pm 0,14$ & 1,52 & $\pm 0,14$ & 0,730 \\
IMC & 18,49 & $\pm 3,75$ & 20,34 & $\pm 2,92$ & 0,120 \\
IDADE & 11,63 & $\pm 1,95$ & 11,58 & $\pm 2,39$ & 0,940 \\
RCE & 0,47 & $\pm 0,08$ & 0,51 & $\pm 0,05$ & 0,179 \\
\hline
\end{tabular}

Legenda: $\mathrm{M}=$ MÉDIA, DP $=$ DESVIO PADRÃO, IMC $=$ ÍNDICE DE MASSA CORPORAL, RCE $=$ RELAÇÃO CINTURA ESTATURA, P = VALOR de significância de $95 \%(\mathrm{p}<0,05)$ resultante do teste t para amostras independentes.

A tabela 02 apresenta a comparação de verificado diferença estatisticamente significativa valores médios dos testes velocidade e agilidade $\quad(\mathrm{p}>0,05)$. entre o sexo masculino e feminino. Não foi

Tabela 02: Comparação do teste de agilidade e velocidade entre os sexos.

\begin{tabular}{lcccccc}
\hline \multicolumn{5}{c}{ MASCULINO } & \multicolumn{2}{c}{ FEMININO } \\
& M & DP & M & DP & P \\
\hline TESTE DE AGILIDADE & 7,32 & $\pm 0,79$ & 7,59 & $\pm 0,39$ & 0,274 \\
CORRIDA 20M & 3,99 & $\pm 0,53$ & 4,07 & $\pm 0,33$ & 0,509 \\
\hline $\begin{array}{l}\text { Legenda: M = MÉDIA, DP = DESVIO PADRÃO, P = VALOR de significância de } 95 \%(\mathrm{p}<0,05) \text { resultante do } \\
\text { teste t para amostras independentes. }\end{array}$
\end{tabular}


A tabela 03 apresenta a associação do risco de resultados. Foi encontrado em maior de desenvolvimento de doenças cardiovasculares, percentual do grupo do sexo masculino o baixo a partir do cálculo do RCE, com o sexo. Nessa risco, enquanto um maior percentual das meninas tabela pode-se ver que houve associação entre os apresentou alto risco para desenvolvimento de gêneros, ou seja, houve diferença na distribuição doenças relacionados ao RCE.

Tabela 03: Associação do risco de desenvolvimento de doenças cardíacas, a partir do RCE, com o sexo.

\begin{tabular}{llclcl}
\hline & \multicolumn{3}{l}{$(>0,5)$} & ALTO RISCO & \multicolumn{3}{l}{$(<0,5)$} & MENOR RISCO & \\
& $\mathbf{N}$ & $\mathbf{\%}$ & $\mathbf{N}$ & $\mathbf{\%}$ & $\mathbf{P}$ \\
\hline \multirow{2}{*}{ MASCULINO } & 11 & $28,9 \%$ & 27 & $71,1 \%$ & \\
& & & & & 0,03 \\
FEMININO & 8 & $66,7 \%$ & 4 & $33,3 \%$ & \\
\hline
\end{tabular}

Legenda: $\mathrm{P}=$ VALOR de significância de $95 \%(\mathrm{p}<0,05)$ resultante do teste de associação Qui-quadrado.

As tabelas 04 e 05 mostram a associação diferenças entre os percentuais dos gêneros não entre o gênero e as classificações dos testes de se apresentaram significativas. agilidade e velocidade. Em ambas as tabelas as

Tabela 04: Associação entre o desempenho no teste de velocidade de 20 metros e os sexos.

\begin{tabular}{|c|c|c|c|c|c|c|c|c|c|}
\hline & \multicolumn{2}{|c|}{ FRACO } & \multicolumn{2}{|c|}{ RAZOÁVEL } & \multicolumn{2}{|r|}{ BOM } & \multicolumn{2}{|c|}{ MUITO BOM } & \multirow[b]{2}{*}{$\mathbf{P}$} \\
\hline & $\mathbf{N}$ & $\%$ & $\mathbf{N}$ & $\%$ & $\mathbf{N}$ & $\%$ & $\mathbf{N}$ & $\%$ & \\
\hline MASCULINO & 17 & $(44,7 \%)$ & 15 & $(39,5 \%)$ & 3 & $(7,9 \%)$ & 3 & $(7,9 \%)$ & \\
\hline FEMININO & 2 & $(16,7 \%)$ & 3 & $(25,0 \%)$ & 5 & $(41,7 \%)$ & 2 & $(16,7 \%)$ & 0,435 \\
\hline
\end{tabular}

Tabela 05: Associação entre o desempenho no teste de agilidade e os sexos.

\begin{tabular}{|c|c|c|c|c|c|c|c|}
\hline & \multicolumn{2}{|c|}{ FRACO } & \multicolumn{2}{|c|}{ RAZOÁVEL } & \multicolumn{2}{|c|}{ ВOM } & \multirow[b]{2}{*}{$\mathbf{P}$} \\
\hline & $\mathbf{N}$ & $\%$ & $\mathbf{N}$ & $\%$ & $\mathbf{N}$ & $\%$ & \\
\hline MASCULINO & 26 & $68,4 \%$ & 10 & $26,3 \%$ & 2 & $5,3 \%$ & \\
\hline FEMININO & 10 & $83,3 \%$ & 2 & $16,7 \%$ & 0 & $0,0 \%$ & 0,521 \\
\hline
\end{tabular}

$\mathrm{P}=$ VALOR de significância de $95 \%(\mathrm{p}<0,05)$ resultante do teste de associação Qui-quadrado. 


\section{DISCUSSÃO}

Mundialmente, o nível de atividade física entre crianças e adolescentes vem se mostrando muito baixo e um dos principais fatores que contribuem para este quadro é o uso por partes das crianças e adolescentes de dispositivos eletrônicos como TV, jogos eletrônicos, celulares entre outros. O comportamento sedentário tem seu foco no tempo de tela, ou seja, a soma do tempo gasto assistindo televisão, jogos eletrônicos e usando um computador ou outros dispositivos eletrônicos ${ }^{(6)}$.

O Departamento de Saúde e Serviços Humanos dos EUA afirma que crianças e adolescentes devem gastar pelo menos 60 minutos diários em atividade física moderada a vigorosa a fim de promover benefício à saúde a curto e longo prazo. Entretanto, uma pesquisa com adolescentes norte-americanos com idades de 12-15, mostrou que apenas cerca de 8\% deste público cumpriam essas recomendações ${ }^{(12)}$.

As atividades físicas recreativas se mostram uma excelente ferramenta para mudar este quadro de atividade física insuficiente entre crianças e adolescentes em todo o mundo. Por isso, projetos sociais esportivos (PSE) e as aulas de educação física escolar se fazem tão importantes.

$\mathrm{O}$ presente estudo mostrou que entre as crianças e adolescentes atendidas no projeto iniciação não foram encontradas diferenças significativas entre meninos e meninas para o índice de massa corpórea (IMC), relação cintura- estatura (RCE), idade, peso e altura. No mesmo sentido, um estudo realizado por Testa, Poeta e Duarte ${ }^{(13)}$, em crianças de 8 a 9 anos de idade, submetidas a um programa de intervenção com exercício físico com atividades recreativas e orientação nutricional durante 12 semanas consecutivas, obteve resultados significativos na diminuição do IMC e do percentual de gordura corporal quando comparado ao grupo controle.

Vale ressaltar que o IMC é um parâmetro adotado pela Organização Mundial de Saúde(OMS) para calcular o peso ideal de cada pessoa. O índice é calculado da seguinte maneira: divide-se o peso da pessoa pela sua altura elevada ao quadrado. O RCE é um indicador de excesso de gordura visceral capaz de predizer fatores de risco com medidas mais acessíveis à população em geral. Além disso, o ponto de corte para identificar a zona saudável e de risco independe das variáveis idade, sexo, etnia e outros.

Em relação aos testes das capacidades físicas de velocidade e agilidade, ambos os sexos tiveram desempenho semelhante. Santos ${ }^{(14)}$, aponta avanço significativo na melhoria da agilidade em intervenções de exercícios físicos com crianças e adolescente com excesso de peso. Em um estudo realizado por Souza, Vieira e Magalhães ${ }^{(15)}$, com 30 crianças com idade entre 09 e 11 anos constatou-se que a média de tempo de execução do teste de velocidade (corrida de 30 metros) dos praticantes do futsal foi de 5,37s, enquanto os alunos que não praticavam nenhuma modalidade esportiva apresentaram a média de 
tempo 6,12s. No teste Shuttle Run, que tinha como objetivo analisar a agilidade, os resultados obtidos pelo grupo dos alunos praticantes do futsal apresentaram a média de 11,11s, já o grupo dos alunos que não praticavam nenhuma modalidade esportiva obtiveram a média de 12,6s. Esses resultados corroboram com outros

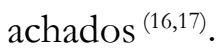

Conceitualmente, pode-se dizer que a velocidade e a agilidades são capacidades físicas que possuem características muito próximas, uma vez que a velocidade pode ser entendida como a capacidade de realizar um movimento no menor espaço de tempo, e a agilidade pode ser definida como a capacidade de realizar uma mudança de direção e de movimento em um menor espaço de tempo. Ambas são valências físicas que possuem algumas particularidades semelhantes. Muitas definições colocam a agilidade como inserida na velocidade, diferenciando-se apenas quanto às mudanças de direção. No entanto a agilidade é uma variável neuro-motora caracterizada pela capacidade de realizar trocas rápidas de direção, sentido e deslocamento da altura do centro de gravidade de todo corpo ou parte dela. Todavia, esses movimentos rápidos fazem da agilidade muito próxima à velocidade, onde ambas atuam de modo proporcionalmente similar ${ }^{(15)}$.

Algo importante a ser relatado é que em nossos estudos foram analisados os parâmetros para IMC, RCE, idade, peso e altura, bem como as valências físicas da velocidade e agilidade de meninos e meninas participante de um projeto social, já nos achados acima citados existia um grupo controle e um intervenção. Entretanto, apesar das diferenças entre as pesquisas é possível afirmar que crianças e adolescentes praticantes de atividades esportivas e recreativas apresentam riscos menores para o desenvolvimento de doenças relacionadas à síndrome metabólica e possuem um maior nível de aptidão física relacionado ao desempenho esportivo.

Os resultados da pesquisa demostraram que a atividade física recreativa é capaz de promover adequação positiva sobre o controle de peso e obesidade em crianças e adolescentes. Contudo, por conta do projeto funcionar somente duas vezes por semana e do baixo número da amostra recomenda-se que pesquisas futuras possam melhorar essa análise usando uma amostra maior e mais diversificada. Os achados deste estudo sugerem ainda que as intervenções do projeto iniciação podem ser eficazes no aumento dos níveis de atividade física de crianças e adolescentes, e esses achados podem auxiliar políticas públicas e educacionais para melhorar os níveis de atividade física e saúde dos mesmos.

\section{CONCLUSÃO}

Concluiu-se que entre as crianças e adolescentes participantes do projeto "iniciação" não houve diferenças significativas quando comparado as capacidades físicas (velocidade e agilidade) e o perfil antropométrico dos participantes. Entretanto, constatou-se um alto risco para o desenvolvimento de doenças 
cardiácas, tendo como parametro o RCE, para o sexo feminino, quando comparado com o grupo do sexo masculino.

\section{Agradecimentos}

Este estudo foi apoiado pelo Conselho Nacional de Desenvolvimento Científico e Tecnológico (CNPq) e Coordenação de Aperfeiçoamento de Pessoal de Nível Superior (CAPES).

\section{REFERÊNCIAS}

[1]Simão JHMN, Poletto L. A importância do lúdico no desenvolvimento do ensino-aprendizagem e motor da criança nos anos iniciais do ensino fundamental. Revista Acadêmica Educação e Cultura em Debate. V 5. N. 1. jan-dez. 2019. Disponível em: http://revistas. unifan.edu.br/index.php/RevistaISE/article/ view/375/301. Acesso em: 03 fev. 2020.

[2]Fernandes LA, Miranda DM, Ribeiro-Silva PC, Pereira DA, Salvador MG, Lage GM. Uma análise do desenvolvimento motor de crianças com transtorno de atenção e hiperatividade (TDAH) . Revista Educação Especial . 2017, 30 (57), 115127. ISSN:1808270X. Disponível em: https://www.redalyc.org/articulo. oa?id=313150464009. Acesso em: 14 fev. 2020.

[3]Scarabel FAG, MORAES JCP. Psicomotricidad, lo lúdico y sus relaciones con la enseñanza en la Educación Inicial: la comprensión de los docentes. Lecturas: Educación Física Y Deportes, 23(245),4962. Disponível em: https://efdeportes.com/index. $\mathrm{php} /$ EFDeportes/article/view/157. Acesso em: 14 fev. 2020

[4]RAMOS RSS. Jogos e brincadeiras como ferramenta de aprendizagem no ensino fundamental. Eventos pedagógicos, v.9, n1, p. 369-384, 2018.

[5]Pedretti Augusto, Seabra A, Pedretti Alessandro. O futebol recreativo como uma nova abordagem terapêutica para a obesidade em crianças e adolescentes: uma revisão sistemática. Rev Bras Ativ Fís Saúde, v. 21, n. 2, p. 123 32, 2016.
[6]Downing KL, Hinkley T, Salmon J , Hnatiuk JA, Hesketh KD . Do the correlates of screen time and sedentary time differ in preschool children? BMC Public Health, 2017, 17:285.

[7]Guthold R, Gretchen US, Leanne MR, Fiona CT. Global trends in insufficient physical activity among adolescents: a pooled analysis of 298 populationbased surveys with 1.6 million participants. Lancet Child Adolesc Health, 2020; 4: 23-35.

[8]Nilsen AKO; Anderssen, SA; Johannessen, K; Aadland, KN; Ylvisaaker, J; Loftesnes, JM et al. Bi-directional prospective associations between objectively measured physical activity and fundamental motor skills in children: a two-year follow-up. International Journal of Behavioral Nutrition and Physical Activity.2020, 17:1

[9]Associação brasileira para o estudo da obesidade e da síndrome metabólica. Diretrizes brasileiras de obesidade. 4. ed. São Paulo: ABESO, 2016. Disponível em: http://www.abeso.org.br/uploads/ downloads/92/57fccc403e5da.pdf . Acesso em: 14 fev. 2020.

[10]Ashwell M, Hsieh SD. Six reasons why the waistto-height ratio is a rapid and effective global indicator for health risks of obesity and how its use would simplify the international public health message on obesity. Int J Food Sci Nutr. 2005;56:303-7.

[11] Gaya Adroaldo, Gaya Anelise Reis. Projeto esporte Brasil PROESP-Br. Manual de testes e avaliação. Porto Alegre-RS, 2016. Disponível em: https:// docplayer.com.br/26200892-Projeto-esporte-brasil. htm. Acesso em: 14 fev. 2020.

[12]Master L, Nye RT, Lee S, Nahmod NG, Mariani S, Hale L, et al. Bidirectional, Daily Temporal Associations between Sleep and Physical Activity in Adolescents. Scientific Reports. 2019, 9:7732.

[13]Testa WL, Poeta LS, Duarte MFS. Exercício físico com atividades recreativas: uma alternativa para o tratamento da obesidade infantil. Revista Brasileira de Obesidade, Nutrição e Emagrecimento. São Paulo. v.11. n.62. p.49-55. Mar./Abril. 2017

[14]Santos ALR. Programas de exercícios físicos: atletismo para crianças com sobrepeso e obesidade. 2015. 80 f. Dissertação (Mestrado em Ciências do Movimento Humano) - Faculdade de Ciências da Saúde, Universidade Metodista de Piracicaba. Piracicaba: UNIMEP, 2015. 
[15]Souza MRV, Vieira AAT, Magalhães F. Análise das diferenças na velocidade e agilidade em crianças praticantes e não praticantes do futsal na cidade de Lago dos Rodrigues - MA. Revista brasileira de assuntos interdisciplinares - REBAI. V. 1, n.1, Jan/ Jul, 2017

[16]Bortoni WL, Bojikian LP. Crescimento e aptidão física em escolares do sexo masculino, participantes de programa de iniciação esportiva. CEP, v. 3470, 2007. p. 130.Disponível em: https://www.redalyc. org/pdf/930/93010404.pdf. Acesso em: 15 fev. 2020.

[17]Passos WS, Alonso L. A influência do treinamento de futsal na velocidade e agilidade de escolares. EFDeportes.com. Revista Digital. Buenos Aires. Núm. 129. 2009.

OBSERVAÇÃO: Os autores declaram não existir conflitos de interesse de qualquer natureza. 